\title{
The Temporal Pattern of Arterial Stiffness during Aging: A Large- Scale Cross-Sectional Study
}

\author{
Zhengli Tang, ${ }^{1}$ Yuanyuan Lu, ${ }^{2}$ Yiming Hao ${ }^{\circ}{ }^{3}$ Robert Morris, ${ }^{4}$ Di Kang, ${ }^{2}$ Fang Wang, \\ Lin Fan, ${ }^{1}$ Weijian Wang $\mathbb{D},{ }^{1}$ Yiqin Wang $\mathbb{D},{ }^{3}$ and Feng Cheng $\mathbb{D}^{2,4}$ \\ ${ }^{1}$ Shuguang Hospital Health Examination Center Affiliated with Shanghai University of Traditional Chinese Medicine, \\ Shanghai 201203, China \\ ${ }^{2}$ Department of Biostatistics and Epidemiology, College of Public Health, University of South Florida, Tampa, FL 33612, USA \\ ${ }^{3}$ Shanghai Key Laboratory of Health Identification and Assessment/Laboratory of Traditional Chinese Medicine Four \\ Diagnostic Information, Shanghai University of Traditional Chinese Medicine, Shanghai 201203, China \\ ${ }^{4}$ Department of Pharmaceutical Sciences, College of Pharmacy, University of South Florida, Tampa, FL 33612, USA
}

Correspondence should be addressed to Weijian Wang; wwjhxj@163.com, Yiqin Wang; wangyiqin2380@sina.com, and Feng Cheng; fcheng1@usf.edu

Received 31 July 2021; Revised 1 November 2021; Accepted 16 November 2021; Published 10 December 2021

Academic Editor: Gaetano Santulli

Copyright (c) 2021 Zhengli Tang et al. This is an open access article distributed under the Creative Commons Attribution License, which permits unrestricted use, distribution, and reproduction in any medium, provided the original work is properly cited.

\begin{abstract}
Brachial-ankle pulse wave velocity (baPWV) is a noninvasive clinical test that provides quantification for the stiffness of both the aorta and peripheral arteries by measuring the brachial and tibial arterial wave velocities. The temporal pattern of baPWV values during aging was investigated in this paper. A gradual increase in baPWV with respect to age was observed, suggesting an increase in the stiffness of arterial vessels as age increases. The $\triangle \mathrm{baPWV}$ value, defined as the absolute value of the difference between bilateral baPWV, also showed a positive correlation with aging. Many underlying physiological conditions such as hyperlipidemia, hypertension, diabetes, and hyperglycemia have previously been shown to elevate baPWV and contribute to the decline of arterial function. The effect of factors including biological sex, blood pressure, and blood glucose levels on the baPWV temporal pattern were also investigated. Between the ages of 18 and 50, men in the study had significantly higher baPWV readings than females of comparable age on average. However, after the age of 50, mean baPWV values increased at a greater rate in females than in males. In addition, blood pressure and blood glucose were shown to be associated with baPWV values. The results will improve existing prediction models for future cardiovascular episodes induced by arterial hardening in different age groups.
\end{abstract}

\section{Introduction}

The incidence of cardiovascular diseases, and their associated social and economic consequences, is currently on the rise throughout the globe. According to the latest report from the American Heart Association, approximately 3 million deaths were attributed to cardiovascular disease in the United States alone and is the leading cause of death [1]. Globally, approximately 17.8 million deaths were attributed to cardiovascular disease, and as a result of aging populations, it is expected to continue increasing [1]. Previous studies have shown that hardening of arterial vessels is associated with an increased risk of cardiovascular complications and dementia [2-5]. Thus, an early screening of discernable aging of the central or peripheral arteries is an important clinical topic.

Brachial-ankle pulse wave velocity (baPWV) was introduced as an economical measurement for evaluating the mechanical state of the wall of arteries, usually referred as "arterial stiffness," or rigidity of the arterial wall. It is a noninvasive clinical test that provides quantification for the stiffness of both the aorta and peripheral artery by measuring brachial and tibial arterial waves [6,7]. Multiple studies 
have demonstrated the effectiveness of baPWV in measuring the elasticity and hardening of arterial vessels and consequently, characterize the cardiovascular health of cohorts $[8,9]$. Large-scale clinical research like the Atherosclerosis Risk in Communities (ARIC) study has used baPWV as an indicator for evaluating arterial stiffness [10]. In addition, pulse wave velocity was found to be associated with other diseases such as frailty. Frailty is a complex physiological state characterized by significant physical and cognitive impairment [11]. A meta-analysis has recently shown a strong correlation between pulse-wave velocity and frailty. Frail individuals exhibit greater PWV readings $[12,13]$. Parenthetically, a measure of baPWV has now been incorporated as an option in routine health examinations.

Multiple studies have demonstrated the predictive capabilities of baPWV testing with regard to potential future cardiovascular events. For example, a meta-analysis derived from a Japanese population of $n=14,673$, of which all participants did not have a history of cardiovascular disease, showed that everyone's standard deviation increase in baPWV (about $385 \mathrm{~cm} / \mathrm{s}$ ) was associated with a $21 \%$ increase in cardiovascular disease (CVD) risk [14]. A literature metaanalysis, of which included a large number of individuals (>50\%) with medical risk factors associated with CVD risk such as hypertension and late-stage renal disease, showed that every $1 \mathrm{~m} / \mathrm{s}$ increase in baPWV was associated with a $12 \%$ increase in CVD risk [14]. An additional study measured the cumulative incidence of major adverse cardiovascular events (MACE) and showed a strong correlation between elevated baPWV and CVD risk [15]. Thus, many studies have corroborated the effectiveness of using baPWV readings as a predictive indicator of future CVDs.

A healthy baPWV index for individuals of all ages is approximately $1800 \mathrm{~cm} / \mathrm{s}$, and an index value greater than this benchmark is associated with an increasingly higher risk of developing cardiovascular disease [16]. Many underlying physiological conditions such as hyperlipidemia, hypertension, diabetes, and hyperglycemia have previously been shown to elevate baPWV and contribute to the decline of arterial function [11-13, 17-20]. Additionally, age and sex have been shown to influence the stiffness of arteries as well as the risk of developing cardiovascular disease [17]. Many factors will contribute to arterial stiffness; however, there is currently no systematic analysis of the association of baPWV with these factors. Previous studies have been limited in their scope and have only analyzed small populations with respect to the association of baPWV with these possible covariates. In addition, those associations at different age groups were not investigated. In this study, we performed a large-scale cross-sectional study composed of more than 6,000 people and analyzed the temporal pattern of baPWV values ranging from young adults to the elderly. In addition, the association between baPWVs and sex, blood pressure, or blood glucose in a multitude of different age groups. Thus, this paper sought to identify the interplay of associations between these possible confounding variables individually with respect to baPWV as a means to fine-tune and improve existing prediction models for future cardiovascular episodes induced by arterial hardening.

\section{Materials and Methods}

2.1. Data Source. Our data was collected from participants (between 18 and 87 years old) who went through health examinations in the Shuguang Hospital affiliated with Shanghai University of Traditional Chinese Medicine from March 2018 to February 2019.

2.2. Ethics Approval. The study was approved by the Ethics Committee of Shanghai University of Traditional Chinese Medicine and was performed in accordance with the Declaration of Helsinki. All the subjects signed informed consent forms verifying consent and compliance.

2.3. Study Design and Data Collection. A retrospective study was performed to investigate the association of physicochemical test parameters and arterial stiffness in adults. All participants underwent anthropometry, health examinations, and blood biochemistry. In addition, the information of each participant's sex, age, and ethnicity were recorded [21].

Anthropometric data were measured using standard methods published by World Health Organization (WHO). The BMI was calculated as body weight divided by height squared $\left(\mathrm{kg} / \mathrm{m}^{2}\right)$. The baPWV was measured by an BP203RPE III instrument (Omron, Co. Ltd.). After the participant rested in the supine position for at least $5 \mathrm{~min}$, a trained investigator wrapped cuffs around both arms and legs and recorded the pulse waveforms from the cuffs simultaneously. The baPWVs were automatically calculated by the instrument. The blood pressure was measured using an HBP9020 instrument (Omron, Co. Ltd.) in a seated position after a 5-minute rest and was recorded as the mean of two different measurements taken within a 1-minute interval. A fasting blood sample was collected from each participant via the antecubital vein in the morning. Glucose (including fasting plasma glucose (FPG) and hemoglobin Alc $(\mathrm{HbAlc})$ ), serum lipids (including total blood cholesterol (TC), triglycerides (TG), low-density lipoprotein cholesterol (LDL-C), and high-density lipoprotein cholesterol (HDLC)), indicators of liver function (including alanine aminotransferase (ALT), aspartate aminotransferase (AST), and gamma-glutamyl transferase $(\gamma-\mathrm{GT}))$, and indicators of kidney function (including serum uric acid (SUA), serum creatinine ( $\mathrm{sCr}$ ), and estimated glomerular filtration rate (eGFR)) were measured in the hospital laboratory according to routine procedures.

2.4. Statistical Analysis. The effect size (Cohen's $d$ value) of all continuous variables (shown in Table 1) was calculated using the effsize package in R. The group differences (shown in Figures 1-3) were calculated using ANOVA with Bonferroni correction for post hoc analysis. All figures were also plotted using the ggplot2 package in R.

\section{Results}

3.1. The Demographic Distribution as well as the Results of Health Examinations and Blood Biochemistry Tests. Table 1 summarizes the demographic distribution as well as the results of a series of health examinations and blood 
TABLE 1: The demographic distribution as well as the results of health examinations and blood biochemistry tests.

\begin{tabular}{|c|c|c|c|c|}
\hline & Total sample & Low baPWV & High baPWV & Effect size (Cohen's $d$ ) \\
\hline \multicolumn{5}{|l|}{ Sex, $n(\%)$} \\
\hline Male & $3728(60.1)$ & $3325(59.5 \%)$ & $403(64.8 \%)$ & \\
\hline Female & $2482(39.9)$ & $2263(40.5 \%)$ & $219(35.2 \%)$ & \\
\hline Age (years), mean (SD) & $47.9(12.7), n=6210$ & $46.1(11.8), n=5588$ & $63.6(9.4), n=622$ & 1.64 \\
\hline Height $(\mathrm{cm})$, mean $(\mathrm{SD})$ & $167.4(8.4), n=6209$ & $167.8(8.4), n=5587$ & $164.5(8.2), n=622$ & 0.39 \\
\hline Weight (kg), mean (SD) & $68.5(12.9), n=6208$ & $68.7(13.1), n=5587$ & $66.9(10.9), n=621$ & 0.15 \\
\hline BMI $\left(\mathrm{kg} / \mathrm{m}^{2}\right)$, mean $(\mathrm{SD})$ & $24.3(3.4), n=6208$ & $24.3(3.5), n=5587$ & $24.6(3.0), n=621$ & 0.10 \\
\hline $\mathrm{SBP}(\mathrm{mmHg})$, mean $(\mathrm{SD})$ & $128.3(18.7), n=6182$ & $125.5(16.4), n=5567$ & $153.9(18.7), n=615$ & 1.62 \\
\hline DBP (mmHg), mean (SD) & $79.3(11.1), n=6182$ & $78.3(10.6), n=5567$ & $87.6(11.7), n=615$ & 0.84 \\
\hline $\mathrm{FPG}(\mathrm{mmol} / \mathrm{L})$, mean $(\mathrm{SD})$ & $5.3(1.3), n=6180$ & $5.2(1.1), n=5564$ & $6.0(1.9), n=616$ & 0.51 \\
\hline HbAlc (\%), mean (SD) & $5.6(0.8), n=4889$ & $5.5(0.7), n=4331$ & $6.1(1.2), n=558$ & 0.54 \\
\hline TG (mmol/L), mean (SD) & $1.7(1.3), n=6182$ & $1.7(1.3), n=5566$ & $1.9(1.4), n=616$ & 0.15 \\
\hline TC (mmol/L), mean (SD) & $5.1(1.0), n=6183$ & $5.1(1.0), n=5567$ & $5.3(1.1), n=616$ & 0.14 \\
\hline LDL-C (mmol/L), mean (SD) & $3.0(0.8), n=5566$ & $3.0(0.8), n=4993$ & $3.0(0.8), n=573$ & 0.06 \\
\hline HDL-C (mmol/L), mean (SD) & $1.3(0.3), n=5565$ & $1.3(0.3), n=4992$ & $1.3(0.3), n=573$ & 0.04 \\
\hline $\operatorname{ALT}(\mathrm{U} / \mathrm{L})$, mean $(\mathrm{SD})$ & $25.0(19.3), n=6163$ & $25.1(19.5), n=5554$ & $24.4(17.6), n=609$ & 0.04 \\
\hline AST (U/L), mean (SD) & $23.5(11.9), n=6163$ & $23.2(11.7), n=5553$ & $25.6(13.1), n=610$ & 0.19 \\
\hline$\gamma$-GT (U/L), mean (SD) & $33.8(32.6), n=6164$ & $33.3(32.1), n=5554$ & $37.9(37.1), n=610$ & 0.13 \\
\hline $\mathrm{sCr}(\mu \mathrm{mol} / \mathrm{L})$, mean $(\mathrm{SD})$ & $73.5(15.9), n=6189$ & $73.2(15.5), n=5572$ & $75.8(19.1), n=617$ & 0.15 \\
\hline sUA $(\mu \mathrm{mol} / \mathrm{L})$, mean $(\mathrm{SD})$ & 360.7 (91.9), $n=6190$ & $359.8(91.7), n=5573$ & $368.8(93.6), n=617$ & 0.10 \\
\hline eGFR (mL/min), mean (SD) & $100.0(17.2), n=5203$ & $100.8(16.9), n=4702$ & $92.3(18.3), n=501$ & 0.48 \\
\hline
\end{tabular}

BMI: body mass index; SBP: systolic blood pressure; DBP: diastolic blood pressure; FPG: fasting plasma glucose; HbA1c: hemoglobin A1c; TC: total blood cholesterol; TG: triglycerides; LDL-C: low-density lipoprotein cholesterol; HDL-C: high-density lipoprotein cholesterol; ALT: alanine aminotransferase; AST: aspartate aminotransferase; $\gamma$-GT: gamma-glutamyl transferase; sUA: serum uric acid; sCr: serum creatinine; eGFR: estimated glomerular filtration rate.

biochemistry tests of the sample population. In addition, the mean value of these parameters was compared between individuals with significant arterial hardening (baPWV value $\geq 1800 \mathrm{~cm} / \mathrm{s}$ ) and members of the control group with a control group (baPWV value $<1800 \mathrm{~cm} / \mathrm{s}$ ) by quantifying Cohen's $d$ effect size. Based on the magnitude of the resulting Cohen $d$ effect size, these parameters can be divided into three groups, large (Cohen's $d \geq 0.8$ ), medium $(0.8>d \geq 0.5)$, and small $(0.5>d \geq 0.2)$ effect size. Large differences were observed between the control and high baPWV groups with regard to age, SBP, and DBP values. Some factors including FPG and HbAlc showed medium differences in effect size between these two groups.

3.2. The Temporal Pattern of baPWV Values. As Figure 4(a) illustrates, a gradual increase in baPWV was observed with respect to age, suggesting an increase in the stiffness of arterial vessels as aging progresses. Cohorts in the youngest age group, 18 to 30 years of age, exhibited an average baPWV of approximately $1200 \mathrm{~cm} / \mathrm{s}$ and showed the lowest variance when compared to all other age groups. At age 50, the rate at which baPWV increases steadily rose, and members of these older demographics exhibited greater variance in their
baPWV values. In the oldest age group, which included cohort members aged 71 or greater, the average baPWV was approximately $1900 \mathrm{~cm} / \mathrm{s}$ with variance concurrently being at its peak.

3.3. The Temporal Pattern of Bilateral baPWV $(\triangle b a P W V)$ Values. The absolute value of differences between left and right baPWV values is referred to as bilateral baPWV $(\triangle \mathrm{baPWV})$. The relationship between age and $\triangle \mathrm{baPWV}$ values was shown in Figure 4(b). Similar to baPWV, the values and variances of $\triangle \mathrm{baPWV}$ increase with respect to age. For individuals in the 18-30 age group, the mean $\triangle \mathrm{baPWV}$ was approximately $60 \mathrm{~cm} / \mathrm{s}$ with a small degree of variance. As age increased, the $\triangle \mathrm{baPWV}$ values and variance nearly doubled in the oldest age group.

3.4. The Temporal Patterns of baPWV in Male and Female Cohorts. In the youngest age demographic, males exhibited higher baPWVs than females in the same age group with males having an average baPWV value approximately equal to $1250 \mathrm{~cm} / \mathrm{s}$ and females having an average of $1100 \mathrm{~cm} / \mathrm{s}$. As indicated in Figure 1, this trend of higher baPWV values in males directly corresponded with age and continued until age 60 , of which then the baPWV values of both males and females began to converge. After 60 , there is no significant 


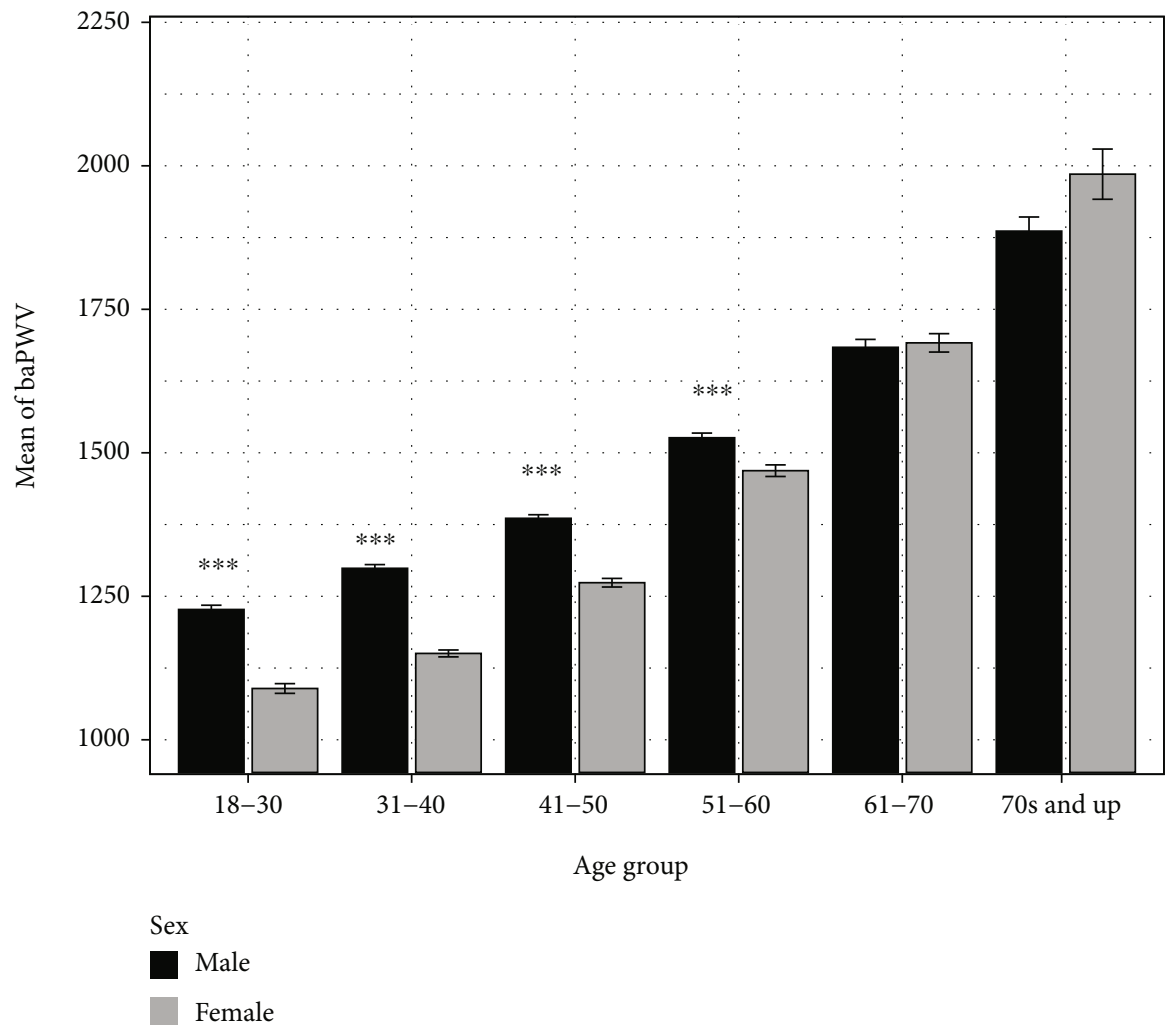

FIgURE 1: The temporal patterns of baPWV in male and female cohorts. The trend of higher baPWV values in males directly corresponded with age and continued until age 60, of which then the baPWV values of both males and females began to converge. At age 50, female cohorts began to show a faster rate of baPWV elevation. Significance level: $P<0.001\left({ }^{* * *}\right), P<0.01\left({ }^{* *}\right)$, and $P<0.05\left({ }^{*}\right)$.

difference in baPWV between males and females. At age $>$ 50 , female cohorts began to display a more rapid stiffening of arteries than males of the same age group as shown by a faster rate of baPWV elevation. Cohorts of age 71 or higher, for both males and females, had average baPWV values greater than the $1800 \mathrm{~cm} / \mathrm{s}$ threshold. In addition, males and females exhibited an increasingly wider variance of baPWV values with respect to age with variance reaching its apex in the oldest age group.

3.5. The Temporal Patterns of baPWV in Cohorts with High or Low Blood Pressure. Figure 2 show the relationship between baPWV and blood pressure (including the systolic blood pressure (SBP) and diastolic blood pressure (DBP)) in each age group. As shown in Figures 2(a) and 2(b), individuals with high SBP (>140 $\mathrm{mmHg}$ ) and DBP (>90 mmHg) values also exhibited significantly heightened baPWV readings across all age groups, which indicates a strong correlation between elevated baPWV and heightened blood pressure. In addition, the difference between the mean baPWV of high blood pressure and low blood pressure cohorts increased with respect to age, with the disparity reaching more than $250 \mathrm{~cm} / \mathrm{s}$ for SBP and $150 \mathrm{~cm} / \mathrm{s}$ for the $\mathrm{DBP}$ in the eldest age group. Despite an increasing trend for blood pressure and baPWV with respect to age, the average baPWV for individuals below the critical threshold for
SBP $(\mathrm{SBP} \leq 140)$ did not exceed a baPWV value of $1800 \mathrm{~cm} / \mathrm{s}$ across all age demographics.

3.6. The Temporal Patterns of baPWV in Cohorts with High or Low Blood Glucose. Figure 3 show the relationship between blood glucose level and baPWV values. The fasting plasma glucose (FPG) test is one of the most prevalent diabetes screening methods currently available and measures blood glucose levels after a period of at least 8 hours of no food consumption. Elevated glucose levels during times of fasting are associated with diabetes. Cohorts with an FPG lower than $6.1 \mathrm{mmol} / \mathrm{L}$ are considered physiologically healthy while individuals with an FPG higher than $6.1 \mathrm{mmol} / \mathrm{L}$ are considered of having some degree of underlying physiological impairment.

As shown in Figure 3(a), in the 18-30 age demographic, the average baPWV value for individuals with an FPG less than $6.1 \mathrm{mmol} / \mathrm{L}$ was approximately $1200 \mathrm{~cm} / \mathrm{s}$. In contrast, members of the same age cohort with an FPG greater than $6.1 \mathrm{mmol} / \mathrm{L}$ had a mean baPWV reading of approximately $1500 \mathrm{~cm} / \mathrm{s}$. Thus, there is a strong association between an individual's blood plasma glucose levels and their degree of stiffened blood vessels with respect to baPWV values. However, the difference in baPWV between the high-FPG group and the low-FPG group is highest in the youngest age group and steadily decreases with respect to aging. In 


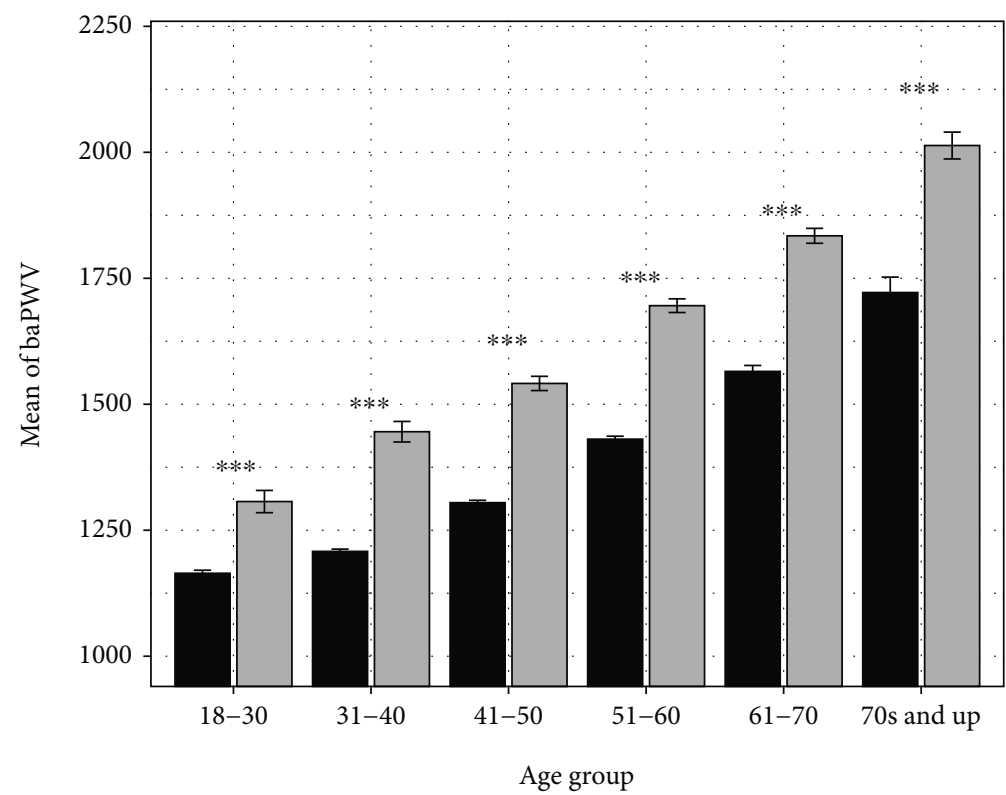

SBP

SBP $<=140$

$\square$ SBP $>140$

(a)

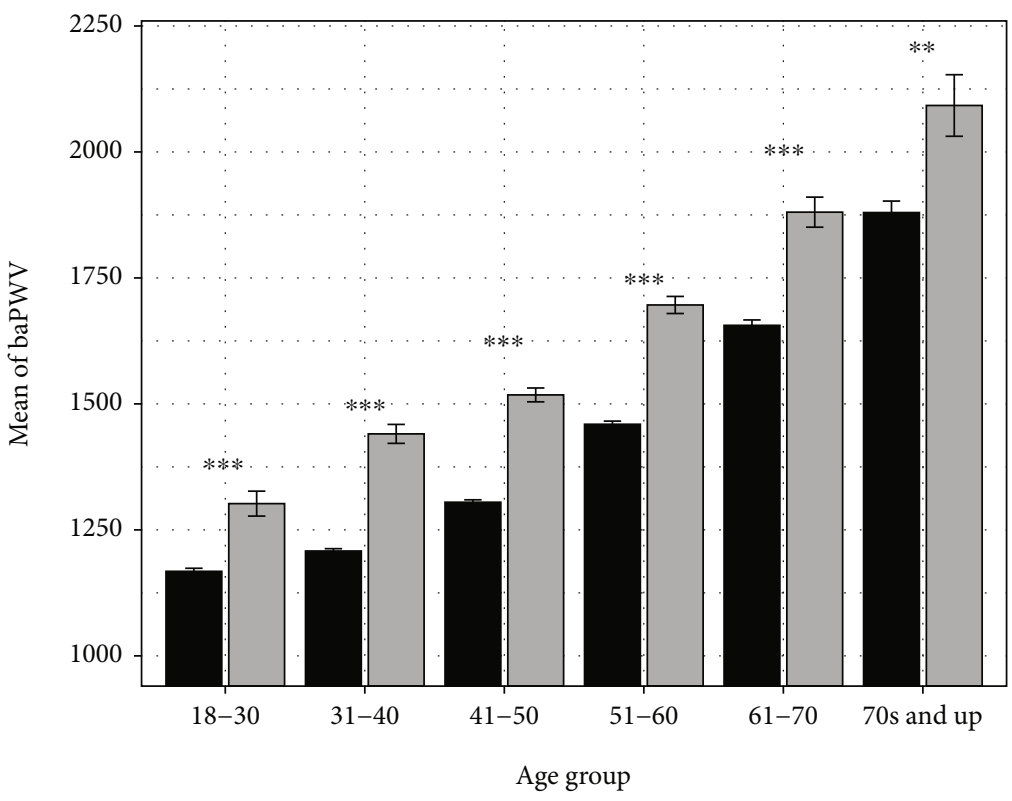

DBP

DBP $<=90$

DBP $>90$

(b)

FIGURE 2: The temporal patterns of baPWV in cohorts with high or low systolic blood pressure (a) and diastolic blood pressure (b). The individuals with raised systolic blood pressure and diastolic blood pressure also exhibited heightened baPWV readings across all age groups. Significance level: $P<0.001\left({ }^{* * *}\right), P<0.01\left(^{* *}\right)$, and $P<0.05\left(^{*}\right)$.

the eldest age demographic, there was no significant difference in mean baPWV between individuals with high FPG and individuals with low FPG values. In addition, individuals in the 18-30 age group with elevated FPG levels had the greatest variance in baPWV readings relative to all other age demographics.

As well as the testing for fasting blood glucose levels, measuring levels of $\mathrm{HbAlc}$, defined as the percentage of 


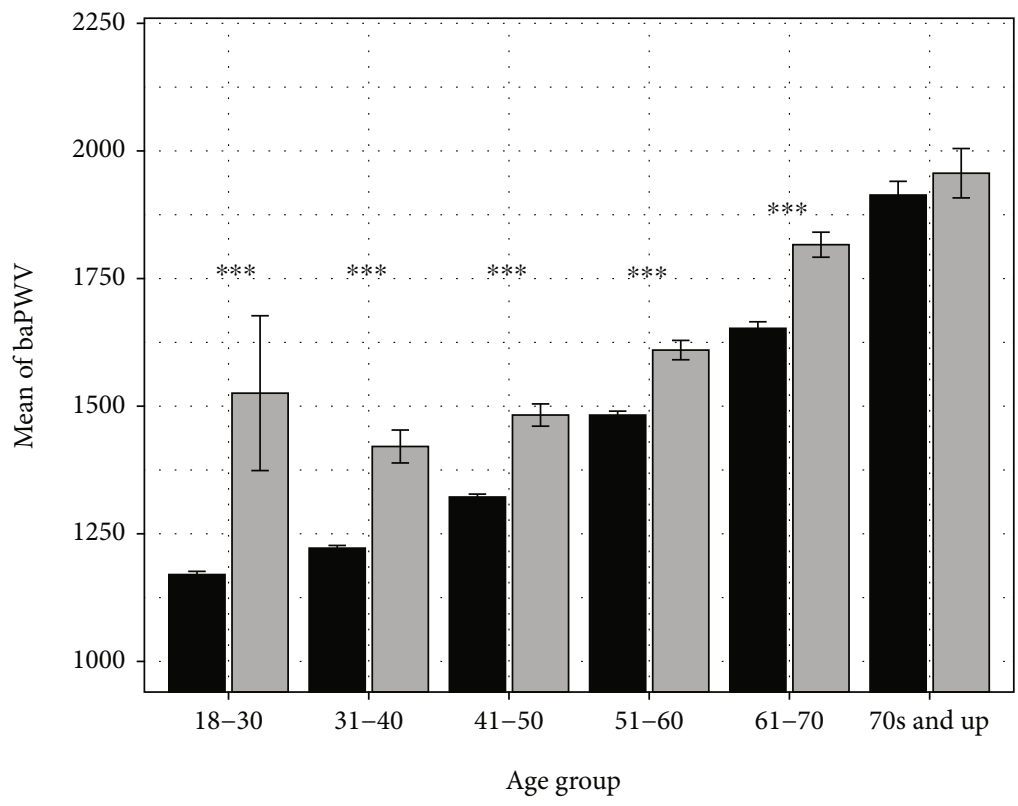

FPG

$\mathrm{FPG}<=6.1$

$\square$ FPG $>6.1$

(a)

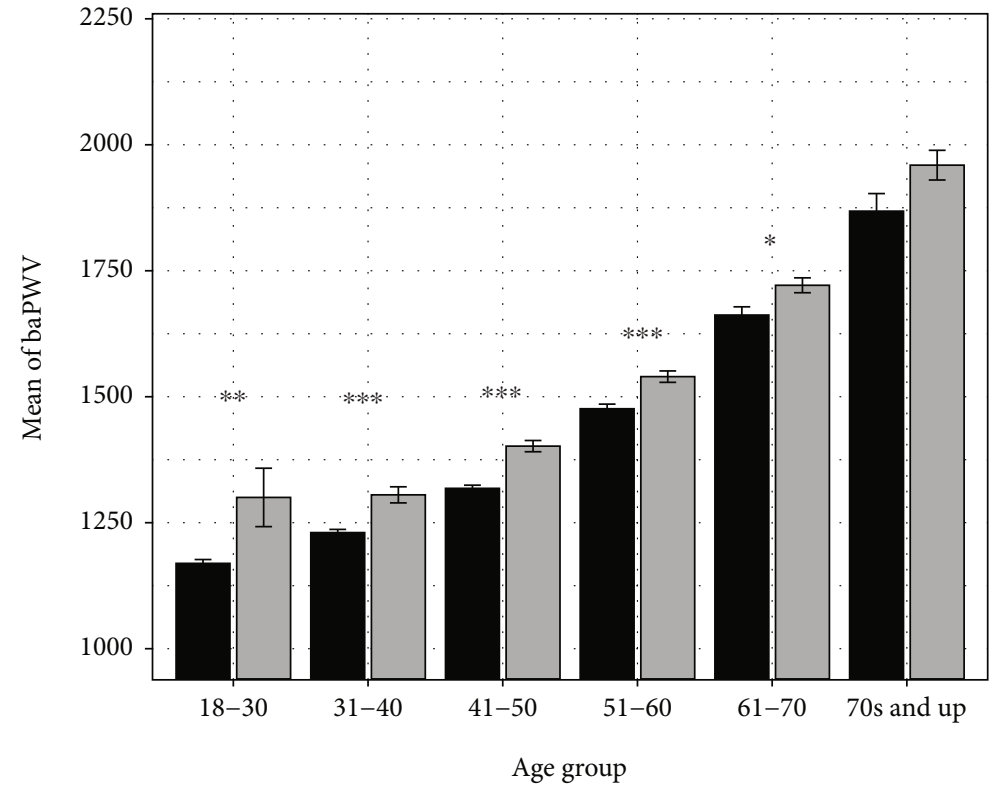

HbA1C

$\mathrm{HbAlc}<=5.6$

$\square \mathrm{HbAlC}>5.6$

(b)

FIGURE 3: The temporal patterns of baPWV in cohorts with high or low fasting plasma glucose (FPG) (a) and hemoglobin A1C (HbA1c) (b). The mean baPWV for individuals with high blood glucose was significantly greater than the corresponding mean baPWV for individuals with low blood glucose with the exception of those in the "70s and up" age group. Significance level: $P<0.001\left({ }^{* * *}\right)$, $P<0.01\left(^{* *}\right)$, and $P<0.05\left({ }^{*}\right)$. 


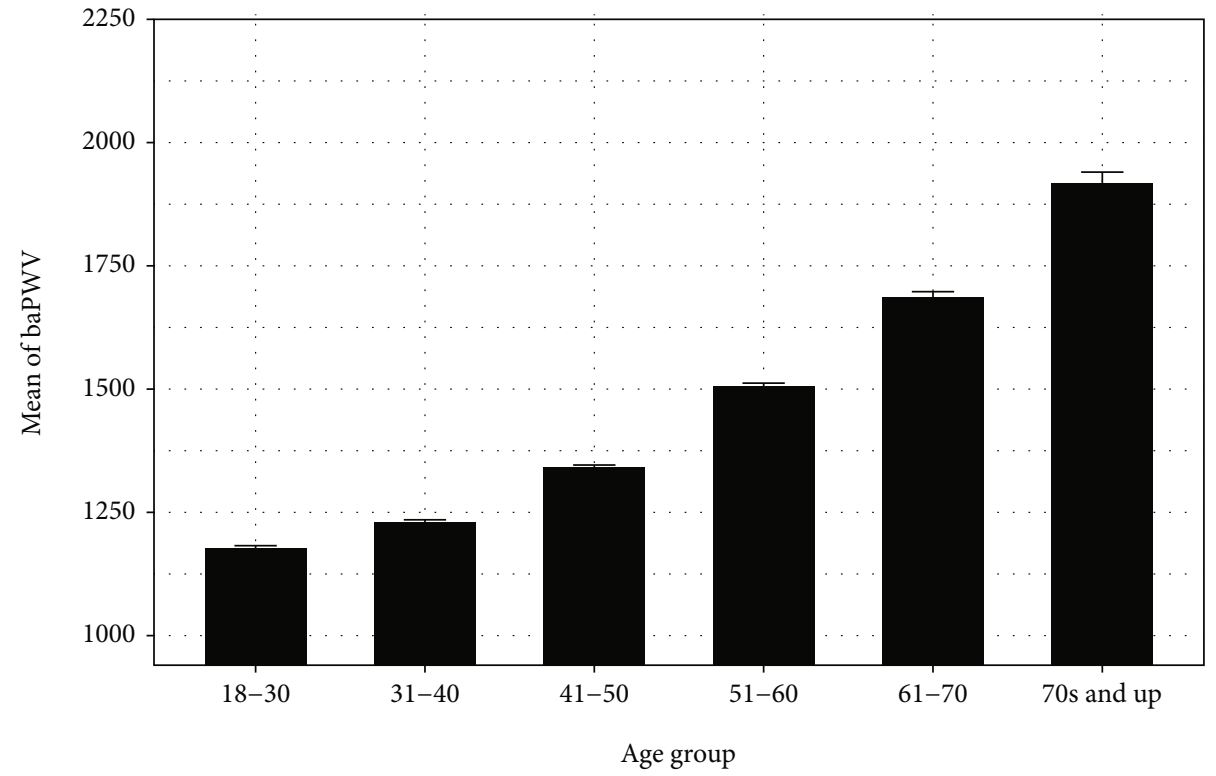

(a)

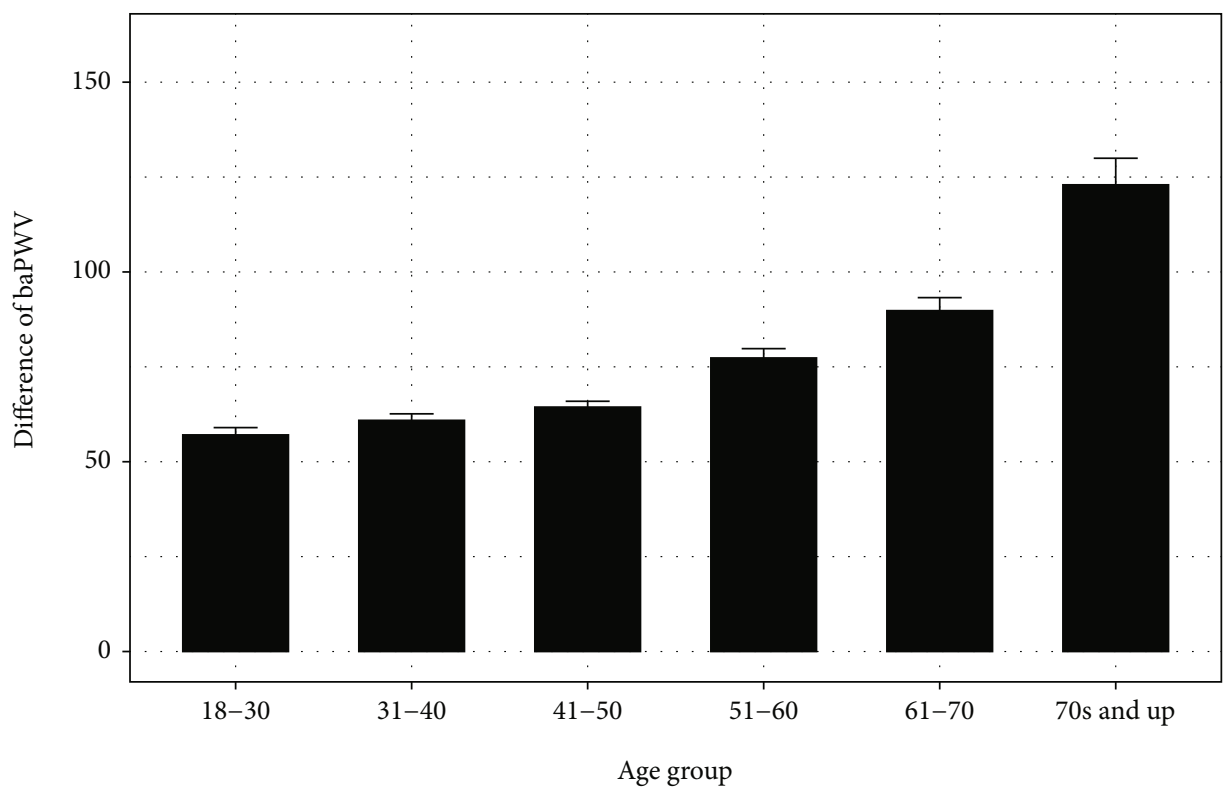

(b)

Figure 4: The temporal pattern of baPWV and bilateral baPWV ( $\triangle \mathrm{baPWV}$ ) values (a) The temporal pattern of baPWV values. A gradual increase in baPWV was observed with respect to age, suggesting an increase in the stiffness of arterial vessels as aging progresses. (b) The temporal pattern of $\triangle \mathrm{baPWV}$ values. The absolute value of differences between left and right baPWV values is referred to as bilateral baPWV ( $\triangle \mathrm{baPWV})$. Similar to baPWV, the values and variances of $\Delta \mathrm{baPWV}$ increase with respect to age.

glucose-bound hemoglobin in the blood, also provides insight into an individual's potential risk for future cardiovascular complications. Healthy individuals are said to have HbAlc levels lower than 5.6\%. HbAlc and baPWV were positively correlated with individuals that have elevated HbAlc (>5.6\%) concurrently having a greater baPWV reading. In the youngest age (age 18-30) demographic, individuals with physiologically healthy $\mathrm{HbA1c}$ levels had a mean baPWV of approximately $1150 \mathrm{~cm} / \mathrm{s}$ while the mean baPWV for individuals with elevated $\mathrm{HbA1c}$ was approximately $1350 \mathrm{~cm} / \mathrm{s}$ (Figure 3(b)). Like FPG (Figure 3(a)), the mean baPWV for individuals with HbAlc $>5.6 \%$ was significantly greater than the corresponding mean baPWV for individuals with $\mathrm{HbAlc} \leq 5.6$ $\%$ with the exception of those in the "70s and up" age group. Individuals in the 18-30 age group with elevated HbA1c levels had the greatest variance in baPWV readings relative to all other age demographics. 


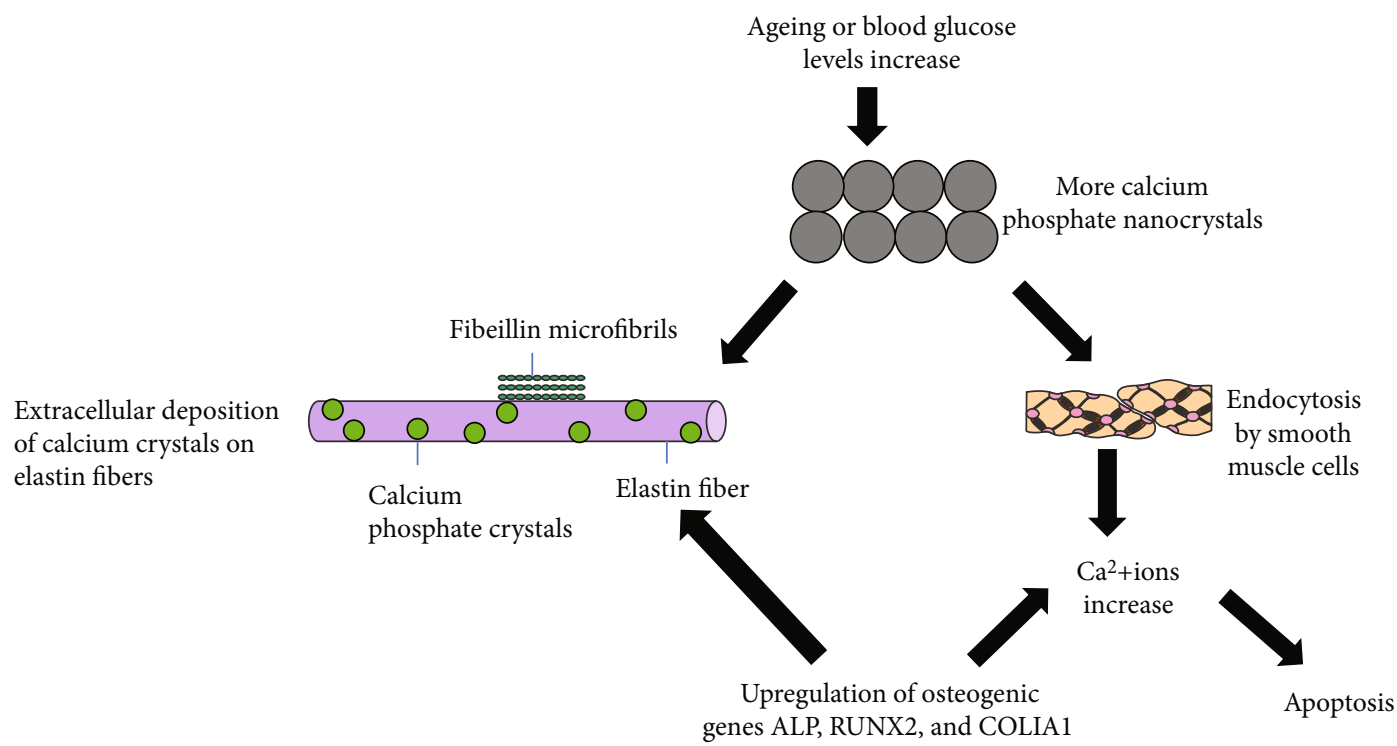

(a)

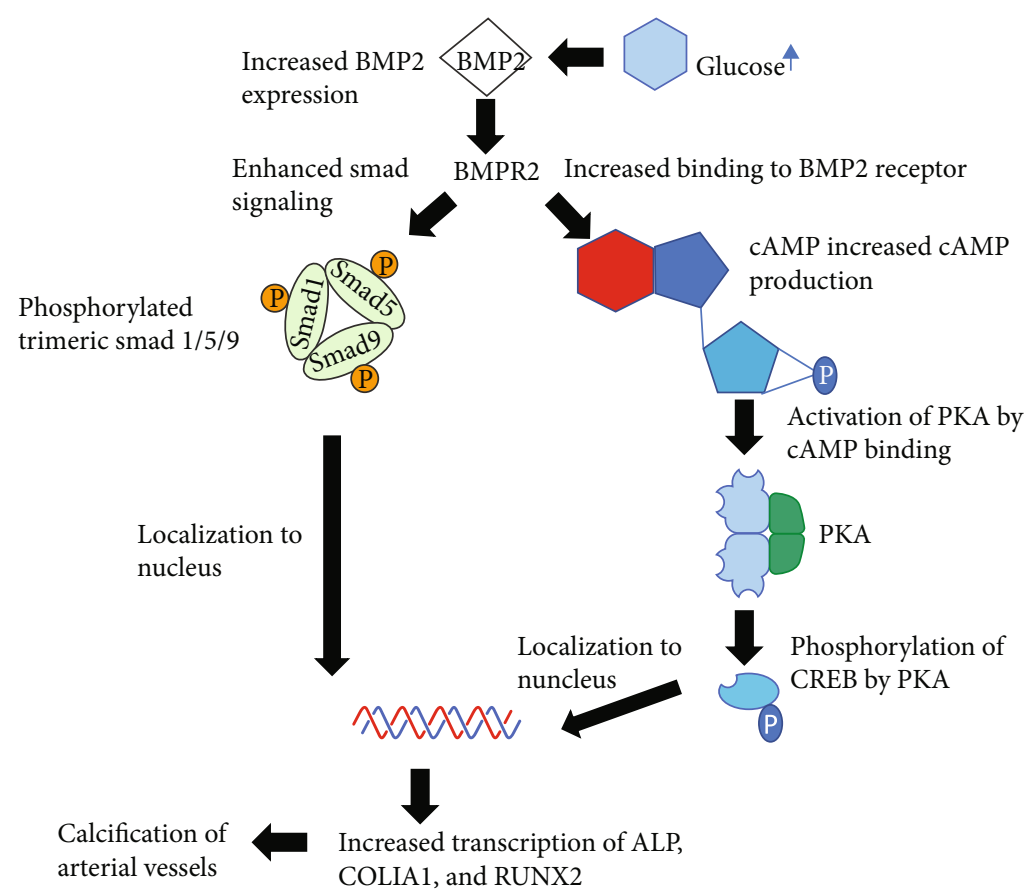

(b)

Figure 5: Two calcification mechanisms of action that drive arterial vessel stiffening. (a) Calcification process of arterial vessels induced by ageing or elevated blood glucose levels. (b) Molecular mechanism in which elevated glucose levels promote calcification and higher baPWV readings.

\section{Discussion}

In this paper, a large-scale cross-section study was performed to identify the temporal pattern of baPWV and bilateral baPWV. The relationship between baPWV and sex, blood pressure, or blood glucose during aging was also investigated.

4.1. The Relationship between Age and baPWV Values. Our results suggested that age is a significant factor associated with artery stiffness with a large effect size (Cohen's $d=$
1.64). Calcification contributes to a more rapid thickening of arterial vessels and subsequently results in the observed elevated baPWV readings during aging [22]. In particular, calcification of coronary arteries (CAC) is influenced by age and sex with approximately $90 \%$ of all men and $67 \%$ of all women over 70 having some degree of CAC. Two mechanisms of calcification (as shown in Figure 5(a)) may promote this loss of elasticity and drive the pathology of stiffened arteries [23]. The first mechanism involves the extracellular deposition of crystallized calcium and amorphous calcium phosphate on elastin fibers, accelerating 
arterial stiffness and promoting inelasticity through formation of stress fractures [22]. The second mechanism promotes accumulation of intracellular calcium in smooth muscle cells via upregulated endocytosis of calcium phosphate [22]. Dysregulated intracellular calcium levels facilitate activation of apoptotic pathways and contribute to loss of arterial vessel integrity through the increase of smooth muscle cell death $[22,24]$. As a result, disrupted calcium metabolism in old age hastens the stiffening of arterial vessels, leading to higher baPWV readings and greater risk of experiencing an adverse cardiovascular event as age increases [24].

However, some studies such as the IKARIA study, of which assessed aortic stiffness and PWV in a small population known for longevity and great health, showed that the age-related increase in arterial stiffness may be remediated by an increase in physical activity and modifications to lifestyle choices $[25,26]$. Other studies have demonstrated an increase in aerobic capacity and a decrease in arterial stiffness in elderly individuals that incorporated moderate physical activity into a previously sedentary lifestyle [27, 28]. Thus, healthy changes in lifestyle choices may protect against the seemingly inevitable age-associated increase in arterial stiffness and dysfunction.

4.2. The Sex Difference in baPWV Values. In this study, men were found on average to have considerably higher baPWV values than women of a comparable age up until the age of 50 , at which point baPWV began to increase at a faster rate in women relative to men. This may coincide with hormonal fluctuations associated with menopausal and postmenopausal physiology and provide an explanation for the more rapid decline in arterial plasticity found in women in their $60 \mathrm{~s}$ and $70 \mathrm{~s}$. Although the exact mechanism is not clear, estrogen has been shown in some clinical studies to have a cardioprotective role in women and helps to modulate agedependent stiffening of arterial vessels [29]. In contrast, testosterone has been shown to have a dual effect in which it may promote cardioprotection in some cases while having deleterious effects on cardiovascular health in others [30]. In males, individuals with low testosterone serum levels have exhibited signs of accelerated arterial aging and increased stiffness through higher carotid-femoral pulse wave velocity values (cfPWV) [30,31]. In populations of men with testosterone deficiency, testosterone supplementation therapy was shown to reverse arterial stiffness to some degree [30]. In females, the significant decline in cardioprotective serum estrogen during and after menopause may, through some yet unelucidated means, promote the anticardioprotective role of testosterone and drive accelerated arterial aging [29, 32]. These hormonal fluctuations may provide insight as to why women experience faster arterial vessel stiffening in old age relative to men of the same age group.

4.3. The Relationship between Blood Pressure and baPWV. Based on the effect size, large differences were observed between the control and high baPWV groups with regard to systolic and diastolic blood pressures. Elevated SBP and DBP values, especially for a prolonged period, exacerbate the deterioration of arterial integrity and increases the rate of arterial aging [33,34]. Healthy blood pressure is maintained by fluctuations in cardiac output and peripheral resistance. However, as one ages, cardiac output declines due to a weakening of healthy cardiomyocyte activity, thus resulting in dysregulation of blood pressure modulation and an increase in mechanical burden on arterial structures [18]. As previously mentioned, prolonged exposure to elevated blood pressure is strongly correlated with an increase in not only cardiovascular disease but also various forms of dementia [35]. One study found a significant increase in $\mathrm{A} \beta$ peptide deposition in nondemented patients over time that had higher baPWV and subsequently, severe hypertension [35]. As $\mathrm{A} \beta$ peptides are thought to contribute to the development of the cognitive deficits observed in dementia pathology [36], increased arterial dysfunction may be associated with cognitive decline.

4.4. The Relationship between Blood Glucose Level and $b a P W V$. In addition to age, sex, and blood pressure, blood glucose levels such as high fasting glucose levels and elevated HbAlc have been shown previously in other studies to be associated with greater baPWV values and higher risk of experiencing an adverse cardiovascular event. In this study, FPG and HbAlc showed medium differences in effect size when assessing their association with baPWV levels.

A recently published observational study $(n=2640)$ following two generations of individuals found a strong correlation between arterial stiffness and markers of hyperglycemia in the parental generation, particularly fasting plasma glucose and HbAlc [37]. Another study suggested that in diabetic patients, high glucose levels were associated with higher baPWV and may promote arterial calcification through an increase in accumulation of intracellular calcium by overstimulation of the BMP-2/RUNX2 signaling pathway $[38,39]$. Figure 5(b) summarizes the possible pathway that may explain how elevated glucose levels contribute to greater baPWV values. Hyperglycemic conditions induce bone morphogenetic protein 2- (BMP-2-) mediated signaling by stimulating phosphorylated trimeric SMAD 1/5/9 proteins as well as promoting cAMP production [40]. Upregulation of cAMP signaling leads to an increase in intracellular calcium accumulation while enhanced SMAD activity leads to the upregulation of osteogenic transcription factors such as runt-related transcription factor 2 (RUNX2), alkaline phosphatase (ALP), and collagen type I alpha 1 (COLIA1) [40]. The upregulation of these genes promotes osteoblastic differentiation, of which RUNX2 is an early biomarker of the osteoblast phenotype and propels the calcification of cardiac cells and vascular smooth muscle cells (VSMCs) $[38,41]$. It has been shown in a previous study that calcification was inhibited in smooth muscle cells exhibiting RUNX2 deficiency, suggesting a role of RUNX2 in arterial stiffness and promoting elevated baPWV [42]. This phenotypic switch in cardiac cells provides a possible explanation as to why high baPWV values are positively associated with elevated glucose levels and why diabetic individuals are more at risk for experiencing an adverse cardiovascular event. 
4.5. Limitations. Despite the novelty of the findings, some limitations are present in the study. For example, despite the large sample size, the relative homogeneity of the sample population may limit the applicability of these findings to other populations to some degree. As is the case with cross-sectional studies, the cause and effect between baPWV and some of the analyzed parameters cannot be assessed. This study cannot say with utmost certainty whether elevated baPWV precedes or follows changes in blood pressure, HbAlc, and FPG. However, based on previous studies and review of the literature, it seems more plausible that increases in baPWV are a byproduct of disruptions in these physiological processes and not an initiator of such disruptions. Further studies will seek to better understand the relationship between the confounding factors with one another as well as to dive deeper into the sex-based differences observed in this study. Transcriptomic data will also be analyzed to better understand and confirm the underlying mechanisms of calcification in adults and how various confounding factors influence this process.

\section{Conclusion}

baPWV is a noninvasive and inexpensive means to estimate arterial thickness and approximate future risk of experiencing a major adverse cardiovascular event. Utilizing electronic medical records derived from the Shuguang Hospital in Shanghai, China, between March 2018 and February 2019, a cross-sectional study was performed to assess the agerelated temporal pattern of baPWV and bilateral baPWV values ranging from young adults to the elderly. In addition, association between baPWV and additional risk factors previously known to influence the possible risk of adverse cardiovascular events such as sex, blood pressure, and blood glucose in a multitude of different age groups. Age, SBP, DBP, FPG, and HbAlc were found to be significantly associated with baPWV readings. Diabetics or prediabetics may have higher baPWV values due to the induction of BMP-2 by elevated glucose levels. Increased transcription of the osteogenic genes ALP, COLIA1, and RUNX2 may promote calcification of arterial vessels, ultimately leading to stiffened arterial walls and an increase in baPWV. Observed sex differences, particularly with regard to the accelerated stiffening of arterial vessels in women after the age of 50, may be a product of hormonal changes that accompany menopausal and postmenopausal states. The significant reduction in the cardioprotective hormone estrogen may facilitate this heightened decay in arterial integrity and function. Our study provides detailed information for the fine-tuning of existing prediction models for future cardiovascular risk induced by arterial hardening in different age groups.

\section{Data Availability}

The Ethics Committee of Shanghai University of Traditional Chinese Medicine limited the measurement data used to support the results of this study in order to protect the privacy of patients. For researchers who meet the criteria for obtaining confidential data, the data of this study can be obtained from Zhengli Tang (e-mail: sgyytzl@163.com).

\section{Conflicts of Interest}

The authors declare that there is no conflict of interests regarding the publication of this paper.

\section{Authors' Contributions}

Robert Morris and Feng Cheng wrote the manuscript text which was revised by Zhengli Tang and Yiming Hao. Feng Cheng, Yiqin Wang, and Weijian Wang helped in the ideas of study. Zhengli Tang, Fang Wang, and Lin Fan collected the whole clinical data. Yuanyuan Lu, Di Kang, and Robert Morris helped in analyzing the data. All authors reviewed the manuscript. Zhengli Tang, Yuanyuan Lu, Yiming Hao, and Robert Morris contributed equally to this study and share first authorship.

\section{Acknowledgments}

This work was supported by the Three Year Action Plan for Strengthening Public Health System in Shanghai (No. GWIV-28), Shanghai Science and Technology Planning Program (No. 21DZ2271000), and Shanghai Education Commission Program (No. 2021 Science and Technology $02-37)$ to Weijian Wang and Yiqin Wang.

\section{References}

[1] S. S. Virani, A. Alonso, E. J. Benjamin et al., "Heart disease and stroke statistics-2020 update: a report from the American Heart Association," Circulation, vol. 141, no. 9, pp. e139e596, 2020.

[2] H. Tanaka, F. A. Dinenno, K. D. Monahan, C. A. DeSouza, and D. R. Seals, "Carotid artery wall hypertrophy with age is related to local systolic blood pressure in healthy men," Arteriosclerosis, thrombosis, and vascular biology, vol. 21, no. 1, pp. 82-87, 2001.

[3] A. Scuteri, A. Benetos, C. Sierra et al., "Routine assessment of cognitive function in older patients with hypertension seen by primary care physicians: why and how-a decision-making support from the working group on 'hypertension and the brain' of the European Society of Hypertension and from the European Geriatric Medicine Society," Journal of Hypertension, vol. 39, no. 1, pp. 90-100, 2021.

[4] A. Scuteri and H. Wang, "Pulse wave velocity as a marker of cognitive impairment in the elderly," Journal of Alzheimer's Disease, vol. 42, Supplement 4, pp. S401-S410, 2014.

[5] J. A. Chirinos, P. Segers, T. Hughes, and R. Townsend, "Largeartery stiffness in health and disease: JACC state-of-the-art review," Journal of the American College of Cardiology, vol. 74, no. 9, pp. 1237-1263, 2019.

[6] J. Huang, Z. Chen, J. Yuan et al., "Association between body mass index (BMI) and brachial-ankle pulse wave velocity (baPWV) in males with hypertension: a community-based cross-section study in North China," Medical science monitor: international medical journal of experimental and clinical research, vol. 25, pp. 5241-5257, 2019. 
[7] P. G. Cunha, J. Cotter, P. Oliveira et al., "Pulse wave velocity distribution in a cohort study: from arterial stiffness to early vascular aging," Journal of hypertension, vol. 33, no. 7, pp. 1438-1445, 2015.

[8] H. Tomiyama, "Arterial stiffness: is this a marker for the current status or future?," Journal of atherosclerosis and thrombosis, vol. 23, no. 9, pp. 1026-1027, 2016.

[9] R. Asmar, A. Benetos, J. Topouchian et al., "Assessment of arterial distensibility by automatic pulse wave velocity measurement. Validation and clinical application studies," Hypertension, vol. 26, no. 3, pp. 485-490, 1995.

[10] M. L. Meyer, H. Tanaka, P. Palta et al., "Correlates of segmental pulse wave velocity in older adults: the Atherosclerosis Risk in Communities (ARIC) Study," American journal of hypertension, vol. 29, no. 1, pp. 114-122, 2016.

[11] P. Mone, J. Gambardella, A. Pansini et al., "Cognitive impairment in frail hypertensive elderly patients: role of hyperglycemia," Cells, vol. 10, no. 8, p. 2115, 2021.

[12] K. Piotrowicz, B. Gryglewska, T. Grodzicki, and J. Gasowski, "Arterial stiffness and frailty - a systematic review and metaanalysis," Experimental Gerontology, vol. 153, article 111480, 2021.

[13] L. Li, H. Li, L. He, H. Chen, and Y. Li, "Study on the relationship between orthostatic hypotension and heart rate variability, pulse wave velocity index, and frailty index in the elderly: a retrospective observational study," Frontiers in cardiovascular medicine, vol. 7, article 603957, 2020.

[14] T. Ohkuma, T. Ninomiya, H. Tomiyama et al., "Brachial-ankle pulse wave velocity and the risk prediction of cardiovascular disease: an individual participant data meta-analysis," Hypertension, vol. 69, no. 6, pp. 1045-1052, 2017.

[15] Y. Ueki, T. Miura, M. Minamisawa et al., "The usefulness of brachial-ankle pulse wave velocity in predicting long-term cardiovascular events in younger patients," Heart Vessels, vol. 32, no. 6, pp. 660-667, 2017.

[16] M. Munakata, "Brachial-ankle pulse wave velocity in the measurement of arterial stiffness: recent evidence and clinical applications," Current hypertension reviews, vol. 10, no. 1, pp. 49-57, 2014.

[17] Z. Liu, Y. Yang, Y. Zhang et al., “Association of brachial-ankle pulse wave velocity and carotid plaque in Chinese hypertensive adults: effect modification by age," Hypertension Research, vol. 43, no. 8, pp. 808-816, 2020.

[18] S. Wu, C. Jin, S. Li et al., "Aging, arterial stiffness, and blood pressure association in Chinese adults," Hypertension, vol. 73, no. 4, pp. 893-899, 2019.

[19] S. Wilson, P. Mone, S. S. Jankauskas, J. Gambardella, and G. Santulli, "Chronic kidney disease: definition, updated epidemiology, staging, and mechanisms of increased cardiovascular risk," Journal of clinical hypertension (Greenwich, Conn.), vol. 23, no. 4, pp. 831-834, 2021.

[20] C. U. Chae, M. A. Pfeffer, R. J. Glynn, G. F. Mitchell, J. O. Taylor, and C. H. Hennekens, "Increased pulse pressure and risk of heart failure in the elderly," JAMA, vol. 281, no. 7, pp. 634-639, 1999.

[21] Z. Tang, M. Pham, Y. Hao et al., "Sex, age, and BMI modulate the association of physical examinations and blood biochemistry parameters and NAFLD: a retrospective study on 1994 cases observed at Shuguang Hospital, China," BioMed Research International, vol. 2019, Article ID 1246518, 7 pages, 2019.
[22] P. Lanzer, M. Boehm, V. Sorribas et al., "Medial vascular calcification revisited: review and perspectives," European heart journal, vol. 35, no. 23, pp. 1515-1525, 2014.

[23] J. Guo, A. Fujiyoshi, B. Willcox et al., "Increased aortic calcification is associated with arterial stiffness progression in multiethnic middle-aged men," Hypertension, vol. 69, no. 1, pp. 102-108, 2017.

[24] Y. Chen, X. Zhao, and H. Wu, "Arterial stiffness: a focus on vascular calcification and its link to bone mineralization," Arteriosclerosis, thrombosis, and vascular biology, vol. 40, no. 5, pp. 1078-1093, 2020.

[25] P. Pietri, C. Vlachopoulos, C. Chrysohoou et al., "Deceleration of age-related aortic stiffening in a population with high longevity rates: the IKARIA Study," Journal of the American College of Cardiology, vol. 66, no. 16, pp. 1842-1843, 2015.

[26] T. J. LaRocca, C. R. Martens, and D. R. Seals, "Nutrition and other lifestyle influences on arterial aging," Ageing research reviews, vol. 39, pp. 106-119, 2017.

[27] D. G. Jakovljevic, "Physical activity and cardiovascular aging: Physiological and molecular insights," Experimental gerontology, vol. 109, pp. 67-74, 2018.

[28] R. J. Spina, T. E. Meyer, L. R. Peterson, D. T. Villareal, M. R. Rinder, and A. A. Ehsani, "Absence of left ventricular and arterial adaptations to exercise in octogenarians," Journal of Applied Physiology, vol. 97, no. 5, pp. 1654-1659, 2018.

[29] J. Wu, P. W. Hadoke, I. Mair et al., "Modulation of neointimal lesion formation by endogenous androgens is independent of vascular androgen receptor," Cardiovascular research, vol. 103, no. 2, pp. 281-290, 2014.

[30] J. J. DuPont, R. M. Kenney, A. R. Patel, and I. Z. Jaffe, "Sex differences in mechanisms of arterial stiffness," British journal of pharmacology, vol. 176, no. 21, pp. 4208-4225, 2019.

[31] C. Vlachopoulos, N. Ioakeimidis, M. Miner et al., “Testosterone deficiency: a determinant of aortic stiffness in men," Atherosclerosis, vol. 233, no. 1, pp. 278-283, 2014.

[32] S. Samargandy, K. A. Matthews, M. M. Brooks et al., "Arterial stiffness accelerates within 1 year of the final menstrual period: the SWAN Heart Study," Arteriosclerosis, thrombosis, and vascular biology, vol. 40, no. 4, pp. 1001-1008, 2020.

[33] H. Y. Lee and B. H. Oh, "Aging and arterial stiffness," Circulation Journal, vol. 74, no. 11, pp. 2257-2262, 2010.

[34] K. Dumor, M. Shoemaker-Moyle, R. Nistala, and A. Whaley-Connell, "Arterial stiffness in hypertension: an update," Current hypertension reports, vol. 20, no. 8, p. 72, 2018.

[35] T. M. Hughes, L. H. Kuller, E. J. Barinas-Mitchell et al., “Arterial stiffness and $\beta$-Amyloid progression in nondemented elderly adults," JAMA neurology, vol. 71, no. 5, pp. 562-568, 2014.

[36] K. Eckhoff, R. Morris, V. Zuluaga, R. Polsky, and F. Cheng, "The association between tau protein level in cerebrospinal fluid and cognitive status: a large-scale analysis of GAAIN database," Brain sciences, vol. 11, no. 7, p. 861, 2021.

[37] S. Taimour, A. Gottsater, A. Jujic, and P. M. Nilsson, "Hyperglycemia and arterial stiffness across two generations," Journal of Hypertension, vol. 39, no. 3, pp. 471-475, 2021.

[38] F. Liu, H. Zhong, J. Y. Liang et al., "Effect of high glucose levels on the calcification of vascular smooth muscle cells by inducing osteoblastic differentiation and intracellular calcium deposition via BMP-2/Cbf $\alpha-1$ pathway," Journal of Zhejiang University SCIENCE B, vol. 11, no. 12, pp. 905-911, 2010. 
[39] E. S. Choi, E. J. Rhee, J. H. Choi et al., "The association of brachial-ankle pulse wave velocity with 30-minute postchallenge plasma glucose levels in korean adults with no history of type 2 diabetes," Korean diabetes journal, vol. 34, no. 5, pp. 287-293, 2010.

[40] N. X. Chen, D. Duan, K. D. O'Neill, and S. M. Moe, "High glucose increases the expression of Cbfal and BMP-2 and enhances the calcification of vascular smooth muscle cells," Nephrology Dialysis Transplantation, vol. 21, no. 12, pp. 3435-3442, 2006.

[41] M. E. Lin, T. Chen, E. M. Leaf, M. Y. Speer, and C. M. Giachelli, "Runx2 expression in smooth muscle cells is required for arterial medial calcification in mice," The American journal of pathology, vol. 185, no. 7, pp. 19581969, 2015.

[42] Y. Sun, C. H. Byon, K. Yuan et al., "Smooth muscle cell-specific runx2 deficiency inhibits vascular calcification," Circulation research, vol. 111, no. 5, pp. 543-552, 2012. 